\title{
Subanesthetic Ketamine Treatment Promotes Abnormal Interactions between Neural Subsystems and Alters the Properties of Functional Brain Networks
}

\author{
Neil Dawson ${ }^{* 1,2,3}$, Martin McDonald ${ }^{2,4}$, Desmond J Higham ${ }^{2,5}$, Brian J Morris ${ }^{1,6}$ and Judith A Pratt ${ }^{1,2,3}$ \\ 'Psychiatric Research Institute of Neuroscience in Glasgow (PsyRING), Glasgow, UK; ${ }^{2}$ Centre for Neuroscience, University of Strathclyde \\ (CeNsUS), Glasgow, UK; ${ }^{3}$ Strathclyde Institute of Pharmacy and Biomedical Science, University of Strathclyde, Glasgow, UK; \\ ${ }^{4}$ Department of Bioengineering, University of Strathclyde, Glasgow, UK; ${ }^{5}$ Department of Mathematics and Statistics, University of \\ Strathclyde, Glasgow, UK; ${ }^{6}$ Institute of Neuroscience and Psychology (INP), College of Medical, Veterinary and Life Sciences, University of Glasgow, \\ Glasgow, UK
}

Acute treatment with subanesthetic ketamine, a non-competitive $N$-methyl-D-aspartic acid (NMDA) receptor antagonist, is widely utilized as a translational model for schizophrenia. However, how acute NMDA receptor blockade impacts on brain functioning at a systems level, to elicit translationally relevant symptomatology and behavioral deficits, has not yet been determined. Here, for the first time, we apply established and recently validated topological measures from network science to brain imaging data gained from ketamine-treated mice to elucidate how acute NMDA receptor blockade impacts on the properties of functional brain networks. We show that the effects of acute ketamine treatment on the global properties of these networks are divergent from those widely reported in schizophrenia. Where acute NMDA receptor blockade promotes hyperconnectivity in functional brain networks, pronounced dysconnectivity is found in schizophrenia. We also show that acute ketamine treatment increases the connectivity and importance of prefrontal and thalamic brain regions in brain networks, a finding also divergent to alterations seen in schizophrenia. In addition, we characterize how ketamine impacts on bipartite functional interactions between neural subsystems. A key feature includes the enhancement of prefrontal cortex (PFC)-neuromodulatory subsystem connectivity in ketamine-treated animals, a finding consistent with the known effects of ketamine on PFC neurotransmitter levels. Overall, our data suggest that, at a systems level, acute ketamine-induced alterations in brain network connectivity do not parallel those seen in chronic schizophrenia. Hence, the mechanisms through which acute ketamine treatment induces translationally relevant symptomatology may differ from those in chronic schizophrenia. Future effort should therefore be dedicated to resolve the conflicting observations between this putative translational model and schizophrenia. Neuropsychopharmacology (2014) 39, 1786-1798; doi:I0.1038/npp.2014.26; published online 5 March 2014

Keywords: ketamine; 2-deoxyglucose imaging; graph theory; network science

\section{INTRODUCTION}

Schizophrenia is a common, chronic psychiatric disorder characterized by positive symptoms (eg, hallucinations), negative symptoms (eg, blunted affect), and cognitive deficits (eg, executive functioning and memory). The disorder involves dysfunction in a number of brain regions including the prefrontal and temporal cortices, the hippocampus (HP), and thalamic regions. Recent data from functional brain imaging studies in schizophrenia have advanced our understanding of how functional interactions between distinct neural subsystems, such as the prefrontal cortex (PFC) and HP (Meyer-Lindenberg et al, 2005; Benetti

*Correspondence: Dr N Dawson, Division of Biomedical and Life Sciences, Faculty of Health and Medicine, Lancaster University, Lancaster LAI 4YQ, UK, Tel: +44 (0)I52 459 4896,

E-mail: n.dawson I@lancaster.ac.uk

Received 5 November 2013; revised 23 January 2014; accepted 23 January 2014; accepted article preview online 4 February 2014 et al, 2009), and between other discrete cortical regions (Kim et al, 2005) are disrupted in the disorder. However, given that these studies often involve a priori selection of the functional interaction of interest, the data may overemphasize the relative importance of these particular interactions while missing others that are biologically important. Therefore, there has been much interest in taking the alternative approach of characterizing disrupted functional interactions between brain regions in the context of complex brain networks, which may provide added insight into those interactions disrupted in schizophrenia (Lynall et al, 2010; Micheloyannis et al, 2006; Bassett et al, 2008; Liu et al, 2008). More recently, these approaches have also been applied to further understand how risk factors for psychiatric disorder impact on structural brain networks ( $\mathrm{Li}$ et al, 2012) and their application to preclinical brain imaging data with translational relevance to the disorder has started to emerge (Bifone et al, 2010; Dawson et al, 2014). 
There is compelling evidence to support a role for altered glutamate system functioning, and $N$-methyl-D-aspartic acid (NMDA) receptor hypofunction in particular, in schizophrenia. For example, recent in vivo ${ }^{1} \mathrm{H}$ magnetic resonance spectroscopy ( ${ }^{1} \mathrm{H}$-MRS) data support altered glutamate and glutamine levels in the brains of schizophrenia patients (De la Fuente-Sandoval et al, 2011; Marsman et al, 2013; Natsubori et al, 2013). Furthermore, both acute and repeated exposure to NMDA receptor antagonists can induce schizophrenia-like symptoms in humans (Cosgrove and Newell, 1991; Krystal et al, 1994) and acute treatment with the NMDA receptor antagonist ketamine exacerbates the symptoms of schizophrenia patients (Lahti et al, 2001; Malhotra et al, 1997). Due to these observations acute, subanesthetic ketamine treatment has become one of the most widely utilized pharmacological manipulations in both humans (Krystal et al, 1994; Morgan et al, 2004; D’Souza et al, 2012) and animals (Nikiforuk et al, 2010; Pitsikas et al, 2008; Roberts et al, 2010; Skoblenick and Everling, 2012) to model translationally relevant behavioral deficits relevant to schizophrenia. For example, in rodents the subanesthetic dose of ketamine used in this study $(30 \mathrm{mg} / \mathrm{kg})$ has been shown to induce deficits in the startle response (as measured by pre-pulse inhibition), hyperlocomotion and deficits in working memory that have translational relevance to schizophrenia (Galci et al, 2008; Irifune et al, 1991; Kos et al, 2006; Miyamoto et al, 2000; Verma and Moghaddam, 1996; Yang et al, 2010). In addition many studies, in both humans (Langsjo et al, 2004; Vollenweider et al, 1997a, 1997b) and animals (Chih-Liang et al, 2011; Dawson et al, 2013), have been dedicated to elucidate the impact of acute, subanesthetic ketamine treatment on regional brain functioning, with PFC hypermetabolism (hyperfrontality) most consistently reported. The paradox of this ketamine-induced hyperfrontality given the hypofrontality characteristic of long-term schizophrenia (Hill et al, 2004) has not been resolved. Surprisingly, despite the widespread use of ketamine treatment as a translational model there is a relative paucity of data on how acute ketamine treatment impacts on the functional interactions between brain regions and the neural subsystems known to be dysfunctional in schizophrenia. Indeed, only recently have efforts begun to elucidate how acute, subanesthetic doses of ketamine impact on functional connectivity between select brain regions (Niesters et al, 2012; Dawson et al, 2013). Furthermore, to our knowledge, no study has considered the impact of subanesthetic ketamine treatment on functional brain network structure or on functional interactions between neural subsytems within the context of complex brain networks. Here, for the first time, we apply topological measures based on the algorithms of network science to functional 2-deoxyglucose (2-DG) autoradiographic brain imaging data to quantitatively define how acute ketamine treatment impacts on functional brain network connectivity on a global, regional, and neural subsystem scale. Given that recent studies have begun to quantify brain network alterations in schizophrenia, the recent transition of these algorithms to preclinical brain imaging data, and the great interest in the application of subanesthetic ketamine treatment as a translational model for the disorder, it is particularly timely to characterize altered brain network structure in this model. Here, we test the hypothesis that acute NMDA receptor hypofunction models the systems level alterations seen in functional brain networks in schizophrenia and gain new, quantitative insight into the true nature of disrupted brain functioning in this translational model through the application of this analytical approach.

\section{MATERIALS AND METHODS}

All experiments were completed using male C57BL/6J mice (aged 8-9 weeks) group housed (5-6 animals per cage) under standard conditions $\left(21^{\circ} \mathrm{C}, 45-65 \%\right.$ humidity, 12-h dark/light cycle (lights on at $0600 \mathrm{~h}$ )). All experimental manipulations were carried out at least 1 week after entry into the facility and all experiments were carried out in compliance with the Animals (Scientific Procedures) Act, UK 1986. Access to food was restricted for $4-5 \mathrm{~h}$ before the semiquantitative ${ }^{14} \mathrm{C}-2-\mathrm{DG}$ imaging protocol to obviate the potential influence of ketamine treatment on plasma glucose levels.

\section{Semiquantitative ${ }^{14} \mathrm{C}-2$-DG Autoradiographic Imaging}

Measurement of local cerebral glucose utilization (LCGU) was initiated $1 \mathrm{~min}$ after the treatment of mice with $30 \mathrm{mg} / \mathrm{kg}$ ketamine (Sigma-Aldrich, UK; in $2 \mathrm{ml} / \mathrm{kg}$ saline, intraperitoneally (i.p.), $n=9)$ or physiological saline $(1.25 \mathrm{ml} / \mathrm{kg}$, i.p., $n=9$ ) in accordance with previously published protocols (Dawson et al, 2011, 2013). Mice were injected with $4.625 \mathrm{MBq} / \mathrm{kg}$ of $\left[{ }^{14} \mathrm{C}\right]-2-\mathrm{DG}$ (Perkin-Elmer, UK) at a steady rate over a 10 - $s$ period before being returned to their home cage. At exactly $45 \mathrm{~min}$ after isotope injection animals were decapitated and a terminal blood sample was collected, by torso inversion, in heparinized weigh boats. The timing of this imaging protocol ensures that the alterations in brain functioning detected align with the maximal behavioral effects of this dose of ketamine (Miyamoto et al, 2000; Yang et al, 2010). The brain was rapidly dissected out intact then frozen in isopentane $\left(-40^{\circ} \mathrm{C}\right)$ and stored at $-80^{\circ} \mathrm{C}$ until sectioning. Blood samples were centrifuged to separate the plasma and aliquots were removed for the determination of plasma glucose $(10 \mu \mathrm{l})$ and ${ }^{14} \mathrm{C}(20 \mu \mathrm{l})$ concentrations by semiautomated glucose oxidase assay (Beckman Glucose Analyser) and liquid scintillation analysis (Packard), respectively.

Frozen brains were sectioned $(20 \mu \mathrm{m})$ in the coronal plane in a cryostat $\left(-20^{\circ} \mathrm{C}\right)$. A series of three consecutive sections were retained from every $60 \mu \mathrm{m}$, thaw mounted onto slide covers and rapidly dried on a hot plate $\left(70{ }^{\circ} \mathrm{C}\right)$. Autoradiograms were generated by apposing these sections, together with precalibrated ${ }^{14} \mathrm{C}$-standards $(40-1069 \mathrm{nCi} / \mathrm{g}$ tissue equivalents; Amersham International, UK) to X-ray film (Kodak, SB-5) for 5 days. Autoradiographic images were analyzed by a computer-based image analysis system $(\mathrm{MCID} / \mathrm{M} 5+)$. The local isotope concentration for each brain region of interest (RoI) was derived from the optical density (OD) of autoradiographic images relative to that of the coexposed ${ }^{14} \mathrm{C}$ standards. Measurements were taken from 66 anatomically distinct brain regions defined with reference to a stereotaxic mouse brain atlas (Paxinos and Franklin, 2001). The rate of metabolism, LCGU, in each RoI was determined as the ratio of ${ }^{14} \mathrm{C}$ present in that region relative to the average ${ }^{14} \mathrm{C}$ concentration in the whole brain 
of the same animal, and from hereon it will be referred to as the ${ }^{14} \mathrm{C}$-2-DG uptake ratio. Whole brain average ${ }^{14} \mathrm{C}$ levels were determined from the average ${ }^{14} \mathrm{C}$ concentration across all sections in which a RoI was measured. Ketamineinduced alterations in overt LCGU were analyzed using Student's $t$-test and significance was set at $p<0.05$, and anatomically discrete brain regions were assumed to represent independent variables (as previously discussed in McCulloch et al, 1982).

\section{Inter-Regional Correlations and Functional Brain Networks}

The inter-regional Pearson's correlation coefficient was used as the metric of the functional association between brain regions generated from the ${ }^{14} \mathrm{C}-2-\mathrm{DG}$ uptake ratios for each RoI across all animals within the same experimental group (ie, either control or ketamine treated). These correlations were Fisher $z$-transformed to give the correlation data a more normal distribution. This resulted in a pair of $\{66 \times 66\}$ correlation matrices, each within-group matrix representing the specific association strength between each of the 2145 possible pairs of regions. From each correlation matrix $(R)$, we derived a binary adjacency matrix $(A)$ where the functional connection between two regions ( $a^{i, j}$ element) was zero if the Pearson's correlation coefficient was lower than the defined threshold $(p|i, j|<T)$ and unity if the coefficient was greater or equal to the defined threshold $(p|i, j| \geqslant T)$. The adjacency matrix can also be represented as an undirected graph $G$, where a line or an edge represents the functional interaction between two brain regions (nodes) if the correlation coefficient exceeds the defined threshold value.

\section{Network Analysis}

Network architecture was characterized at the global, divisional and regional scales. Global network architecture was quantified in terms of the mean degree $(<k>)$, average path length $\left(L_{\mathrm{p}}\right)$, and mean clustering co-efficient $\left(C_{\mathrm{p}}\right)$ of the whole brain network, as previously described in Dawson et al (2014) and outlined in the section Global Network Architecture. Alterations in the divisional architecture were determined in terms of altered functional clustering, identified in the brain networks through application of the generalized singular value decomposition (GSVD) algorithm (Dawson et al, 2014; Xiao et al, 2011), that provides a data-driven approach to identify clustering difference between the two experimental matrices. Regional properties were defined in terms of degree $\left(k_{i}\right)$, betweenness $\left(B_{\mathrm{c}}\right)$, and closeness $\left(C_{\mathrm{c}}\right)$ centrality. Global and regional metrics were determined on the binary adjacency matrices generated over a range of correlation thresholds (Pearson's $r, T=0.3-$ 0.4 and Fisher's $z, T=0.310-0.424$ ) that were selected on the basis that the maximum threshold utilized yielded fully connected networks in each treatment group and was similar to that utilized in previous reports (Dawson et al, 2014; Liu et al, 2008; Micheloyannis et al, 2006).

\section{Global Network Architecture}

Here we provide brief, formal definitions of the global network metrics determined in this study.
The degree of a node $(k)$ is simply the number of edges that connect that node to the network, so highly connected nodes have a high degree. The mean degree $(<k>$, equation 1$)$ is the average number of edges across all nodes. A sparse network therefore has a low mean degree.

$$
\langle K\rangle=\frac{1}{N} \sum_{i \in G} k_{i}
$$

The minimum path length between two nodes in a graph $\left(L_{i, j}\right)$ is the smallest number of edges that must be traversed to make a connection between them. If two nodes are immediate neighbors, directly connected by a single edge, then $L_{i, j}=1$. The average path length ( $L_{\mathrm{p}}$, equation 2$)$, or average $L_{i, j}$ across all possible pairs, is the average number of steps along the shortest paths across the network. This provides a measure of global network efficiency, where networks with a low average path length are more efficient.

$$
L_{\mathrm{p}}=\frac{1}{2 N(N-1)} \sum_{i j} L_{i j}
$$

The clustering coefficient of node $i\left(\mathrm{C}_{i}\right)$ is the ratio of the number of edges between neighbors of node $i$ relative to the maximum possible number of connections between them. It provides an indication of how well connected the neighborhood of a node is. The mean clustering coefficient $\left(C_{\mathrm{p}}\right.$, equation 3 ) is the average clustering coefficient of all of the nodes in the network, which provides a measure of the local density or cliquishness of the network. A high mean clustering coefficient suggests high clustering and so efficient local information transfer.

$$
C_{p}=\frac{1}{N} \sum_{i \in G} C_{i}
$$

The significance of ketamine-induced alterations in the global properties of 2-DG functional brain networks was determined by comparison of the real difference in each measure with that of networks generated from 1000 to 5000 random permutations of the raw 2-DG data at each correlation threshold (11000-55 000 random permutations in total). Significance was set at $p<0.05$ and was determined from the average $p$-value across the entire correlation threshold range analyzed.

\section{Regional Centrality and Hub Region Identification}

In this study, we consider node centrality as determined by degree $\left(k_{i}\right)$, betweenness $\left(B_{\mathrm{c}}\right)$, and closeness $\left(C_{\mathrm{c}}\right)$. Degree centrality $\left(k_{\mathrm{i}}\right)$ simply measures the number of nodal connections. Betweenness centrality $\left(B_{c}\right.$, equation 4$)$ is based upon how many short paths go through a given node and for the $i$ th node in a graph $(G)$ is defined by:

$$
B_{\mathrm{C}}(i)=\sum_{\substack{i \neq t \in G \\ s \neq t}} \frac{\sigma_{s t}(i)}{\sigma_{s t}}
$$

Here, $\sigma_{s t}$ denotes the number of shortest paths from node $s$ to node $t$, and $\sigma_{s t}(i)$ denotes the number of these that involve node $i$.

Closeness centrality $\left(C_{\mathrm{c}}\right.$, equation 5$)$ is based upon the mean geodesic distance of a node to all other reachable 
nodes in the network, given by:

$$
C_{\mathrm{C}}(i)=\frac{1}{\sum_{t \in G \backslash i} \mathrm{~d} G(i, t)}
$$

Here, $\mathrm{dG}(i, t)$ denotes the geodesic between nodes $i$ and $t$. A brain region that is considered to be an important hub region in the network has a high degree, betweenness, or closeness centrality. In this study, a brain region was defined as a hub in the brain networks of ketamine-treated or control (saline-treated) animals if, for any centrality measure, the regional centrality measure in the real network relative to that of calibrated random Erdös-Rényi graphs (1000 random graphs at each correlation threshold and 11000 random graphs in total) was $z>1.96$. The $z$-score for each centrality measure was calculated as illustrated in equation (6) for the case of betweenness centrality $\left(B_{c}\right)$

$$
Z B_{\mathrm{C}}(i)=\frac{B_{\mathrm{C}}^{\text {real }}-B_{\mathrm{C}}^{\text {random }}}{S D\left(B_{\mathrm{C}}^{\text {random }}\right)}
$$

The significance of ketamine-induced alterations in regional centrality was determined by comparison of the $z$-score difference in regional centrality in the real brain networks with that in networks generated from 11000 random permutations of the raw 2-DG data (1000 random permutations at each correlation threshold). Significance was set at $p<0.05$ with post hoc Bonferroni correction applied to adjust for multiple comparisons on the basis of comparing 66 RoI.

\section{Divisional Architecture; Bipartite Neural Subsystem Interactions}

Differences in the divisional architecture of the functional brain networks between experimental groups were characterized through the application of a recently developed and validated algorithm for cluster identification based upon GSVD. The functionality of this algorithm has previously been proposed, justified, and validated through its application to both synthetic and real biological data sets (Xiao et al, 2011), and has previously been applied to preclinical brain imaging data (Dawson et al, 2014). In the context of this study, the aim of applying this algorithm to the data was to quantitatively define the functional interactions between two defined neural subsystems (bipartite interactions) that are present in one of the experimental groups but not in the other. The ability of the algorithm to elucidate mutually exclusive clusters in two matrices has previously been validated through its application to synthetic data sets (Xiao et al, 2011).

In essence this algorithm allows the re-ordering of two square, symmetric, real-valued matrices $A$ and $B$ with the aim of discovering a new node (brain region) ordering that reveals clusters of nodes that exhibiting strong functional connectivity (mutual weights) in one network but not in the other. In the case of our data, matrices $A$ and matrix $B$ are comprised of the real-value inter-regional correlation coefficients (Pearson's coefficient Fisher transformed) for the control and ketamine treatment group's, respectively. These matrices are identical to those used for the generation of the binary adjacency matrices used to characterize the global and regional characteristics of the functional brain networks in these experimental groups. Once the matrices have been reordered through this iterative algorithm we assessed whether the variance of the node set for a given pair of defined neural subsystems (defined neural subsystems are shown in Figure 4) in the reordered matrix was significantly lower than expected by chance. To determine this, the variance of the nodes for the two neural subsystems of interest in the real GSVD reordered networks was compared with that of a set of nodes equal in size to that of the defined neural subsystem in matrices derived from 10000 random permutations of the raw data. The $p$-values gained from this permutation analysis were corrected by post hoc Bonferroni-Holm correction to adjust for multiple comparisons (110 neural subsystem comparisons made in the control and ketamine-treated data sets (55 bipartite subsystems in each experimental group)). Significance was set at $p<0.05$.

\section{RESULTS}

\section{Ketamine-Induced Alterations in Overt Cerebral Metabolism}

Acute ketamine treatment produced both significant increases and decreases in cerebral metabolism on a region-dependent basis, consistent with previous reports (Dawson et al, 2013; Duncan et al, 1999). Significant overt alterations in cerebral metabolism were identified in 32 of the 66 regions analyzed (Supplementary Table S1A-D); select regions shown in Figure 1). In particular, acute ketamine treatment induced hyperfrontality, an increased rate of cerebral metabolism in subfields of the PFC, an effect that was evident in all subfields of the prelimbic cortex (anterior prelimbic (PrL) and medial prelimbic (layers 1, 2, and 3 (mPrL1, mPrL2, and mPrL3)). In addition, a significant hypermetabolism was found in the entorhinal cortex (EC) and subfields of the HP (molecular layer (ML) and subiculum (Sub)), the nucleus accumbens core (NacC), substantia nigra pars compacta (SNC), and the mamillary body $(\mathrm{MB})$. By contrast, acute ketamine treatment induced a significant thalamic hypometabolism (anteroventral (AVthal), mediodorsal (MDthal), ventrolateral (VLthal), and dorsal reticular (dRT)), along with hypometabolism in the raphé (dorsal (DR), median (MR), and paramedian (PMR)), tegmental (dorsal (DTg) and ventral (VTg)) and habenula (medial (mHab) and lateral (lHab)) nuclei. In addition, ketamine treatment induced a significant hypometabolism in the locus coeruleus (LC), the diagonal band of broca (horizontal (HDB) and vertical (VDB)), medial geniculate (MG), inferior colliculus (IC), the CA3 subfield of the HP and in the retrosplenial cortex (RSC).

\section{Ketamine-Induced Alterations in Global Functional Brain Network Topology}

Acute ketamine treatment resulted in a functional brain network with increased connectivity, as evidenced by the significant increase in mean degree $(\langle k\rangle, p=0.0490)$, the average number of functional connections each brain region (node) has in the network, across the entire correlation threshold range (Figure 2a). However, while the number of connections in the brain network was significantly increased by ketamine treatment this did not significantly 

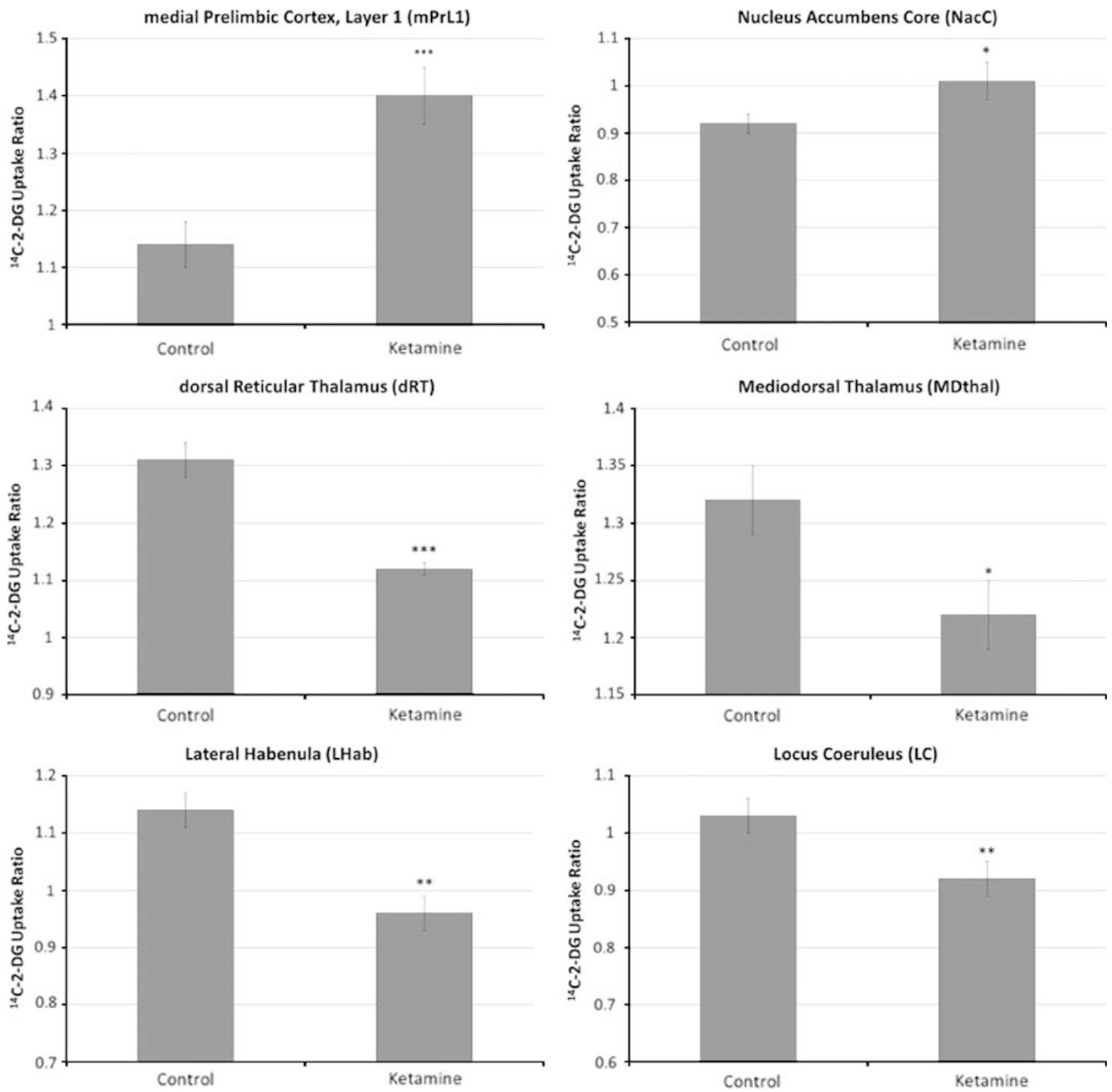

Figure I Ketamine-induced alterations in overt local cerebral glucose utilization (LCGU). Data shown as mean \pm SEM. *Denotes $p<0.05, * *$ denotes $p<0.01$, and *** denotes $p<0.001$ significant difference from control (saline treated) animals ( $t$-test). Full data for all brain regions analyzed are shown in Supplementary Table SIA-D.

impact on average path length $\left(L_{\mathrm{p}}\right.$, Figure $\left.2 \mathrm{~b}, p=0.207\right)$, across the observed correlation threshold range. A low average path length is indicative of a network organized for efficient information transfer on a global scale. The observation that ketamine does not alter $L_{\mathrm{p}}$, thus does not alter the efficiency of information transfer across the network, is surprising given the increased number of functional connections (mean degree, Figure 2a) seen in the brain networks of these animals, and suggests that the efficiency of information transfer across the network on a global scale is not modified by acute ketamine treatment. By contrast, acute ketamine treatment induced a significant increase in the mean clustering coefficient $\left(C_{p}\right.$, Figure $2 c$, $p=0.0333$ ), a measure of local connectivity or cliquishness in the brain network, across the entire correlation threshold range analyzed. This indicates that acute ketamine treatment induces a topological reorganization of the functional brain network resulting in increased efficiency for localized information transfer. When brain networks were considered at the same cost, in essence comparing the global properties of the functional brain networks when they have the same number of functional connections (by allowing weaker functional connections in control animals to be considered as connected) there was no significant difference in the average path length $\left(L_{\mathrm{p}}\right.$, Figure $\left.1 \mathrm{~d}, p=0.0987\right)$ or the mean clustering coefficient $\left(C_{\mathrm{p}}\right.$, Figure $\left.1 \mathrm{e}, p=0.1448\right)$ of the brain 


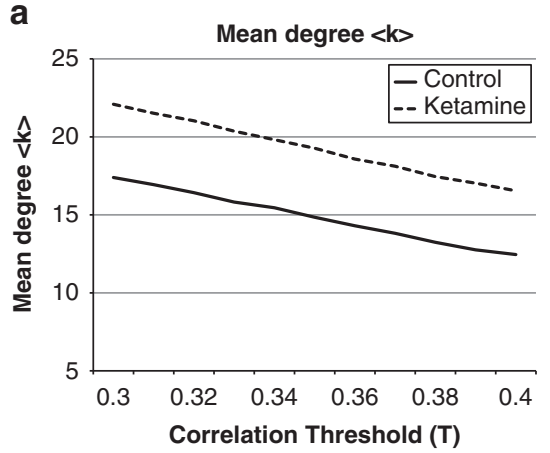

b

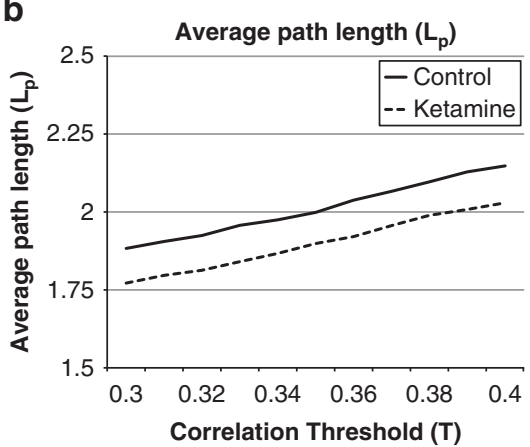

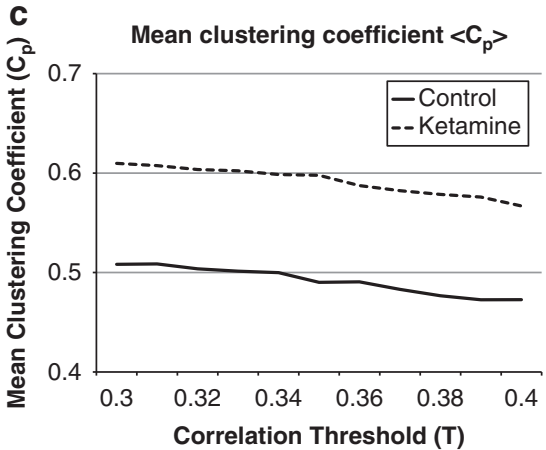
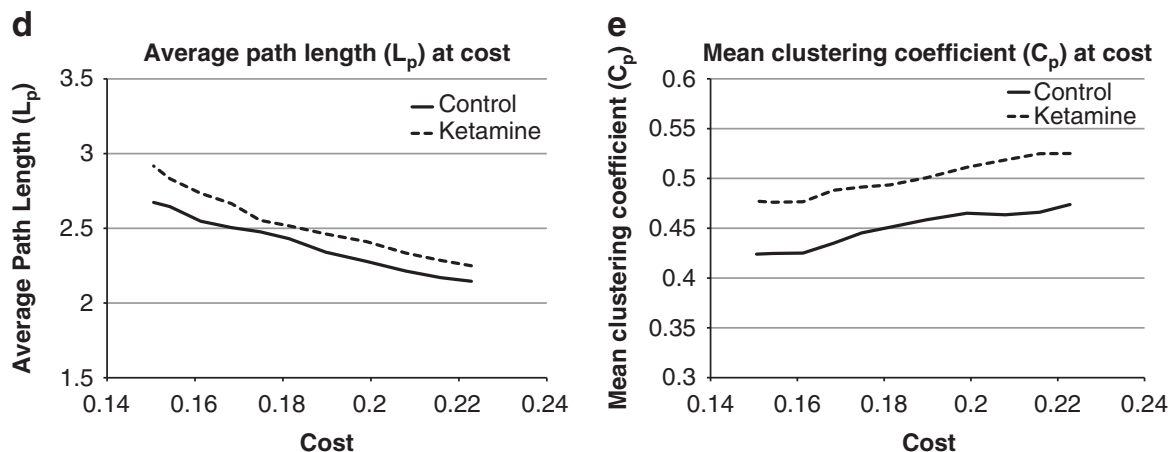

Figure 2 Ketamine-induced alterations in global functional brain network topography. Acute ketamine treatment ( $30 \mathrm{mg} / \mathrm{kg})$ resulted in functional brain networks that displayed a significantly increased $(a)$ mean degree $(p=0.0490)$, had a similar $(b)$ average path length $(p=0.207)$ but a significantly increased (c) mean clustering coefficient ( $p=0.0333$ ) in comparison with networks in control (saline treated) animals. When the functional brain networks of each experimental group were compared at the same cost there was no significant difference between the two groups in terms of the (d) average path length $(p=0.0987)$ or mean clustering coefficient $(p=0.1448)$. The significance of ketamine-induced alterations in global network measures was analyzed by comparison with that in networks generated from $5000(\mathrm{a}-\mathrm{c})$ or $1000(\mathrm{~d}, \mathrm{e})$ random permutations of the raw experimental data. Significance was set at $p<0.05$.

networks. This suggests that the enhanced efficacy of functional connections between brain regions, as indicated by a significantly increased mean degree $(<k>)$, that results from ketamine treatment underlies the increased mean clustering coefficient $\left(C_{\mathrm{p}}\right)$ seen in the brain networks of these animals.

\section{Ketamine-Induced Alterations in Regional Functional Connectivity}

In the functional brain networks of control (saline treated) animal's 4 of the 66 brain regions analyzed were identified as important hubs by centrality analysis (Figure 3; Table 1) including the RSC, nucleus accumbens shell (NacS), interpeduncular nucleus (IP), and MB. In the functional brain network of ketamine-treated animals an increased number of brain regions (14 out of 66) were identified as important hubs. This included two subfields of the PFC (lateral orbital (LO) and mPrL3), four thalamic nuclei (anteromedial thalamic nucleus (AMthal), MDthal, ventromedial thalamus (VMthal), and the centrolateral thalamus (CLthal)), and two amygdala nuclei (medial amygdala (meA) and basolateral amygdala (BLA)). There was little overlap in the identity of brain regions identified as important hubs in the functional brain network of ketamine-treated and control animals, with only the NacS being identified as an important hub in the functional brain network of both experimental groups. This suggests that the organization of the functional brain network is markedly altered by ketamine treatment. Indeed, a statistically significant increase in regional centrality was found for two PFC subfields (mPrL3 and LO), three thalamic nuclei (MDthal, VMthal and CLthal), two amygdala nuclei (MeA and BLA) and the mHab (Table 1). Therefore, these brain regions show significantly increased connectivity and importance in the brain networks of ketamine-treated animals. By contrast, ketamine treatment induced a significant reduction in regional centrality for the RSC and $M B$, suggesting that these hub brain regions in the brain network of control animals were less important and showed significantly reduced connectivity in the brain networks of ketamine-treated animals (Table 1).

\section{Ketamine-Induced Alterations in Bipartite Neural Subsystem Interactions}

GSVD analysis, allowing for the identification of bipartite neural subsystem interactions, revealed that in control animals the thalamus was significantly functionally coupled to both the HP $(p=0.043)$ and the neuromodulatory nuclei $(p=0.011$, Figure 4$)$. The functional interactions between these neural subsytems were not significant in ketaminetreated animals. Rather, GSVD analysis revealed a significant interaction between the neuromodulatory nuclei and the PFC $(p=0.043)$ and other cortical regions (cortex subsystem, $p=0.022$ ) in ketamine-treated animals that was not significantly present in controls. In addition, a significant functional interaction between the thalamus 

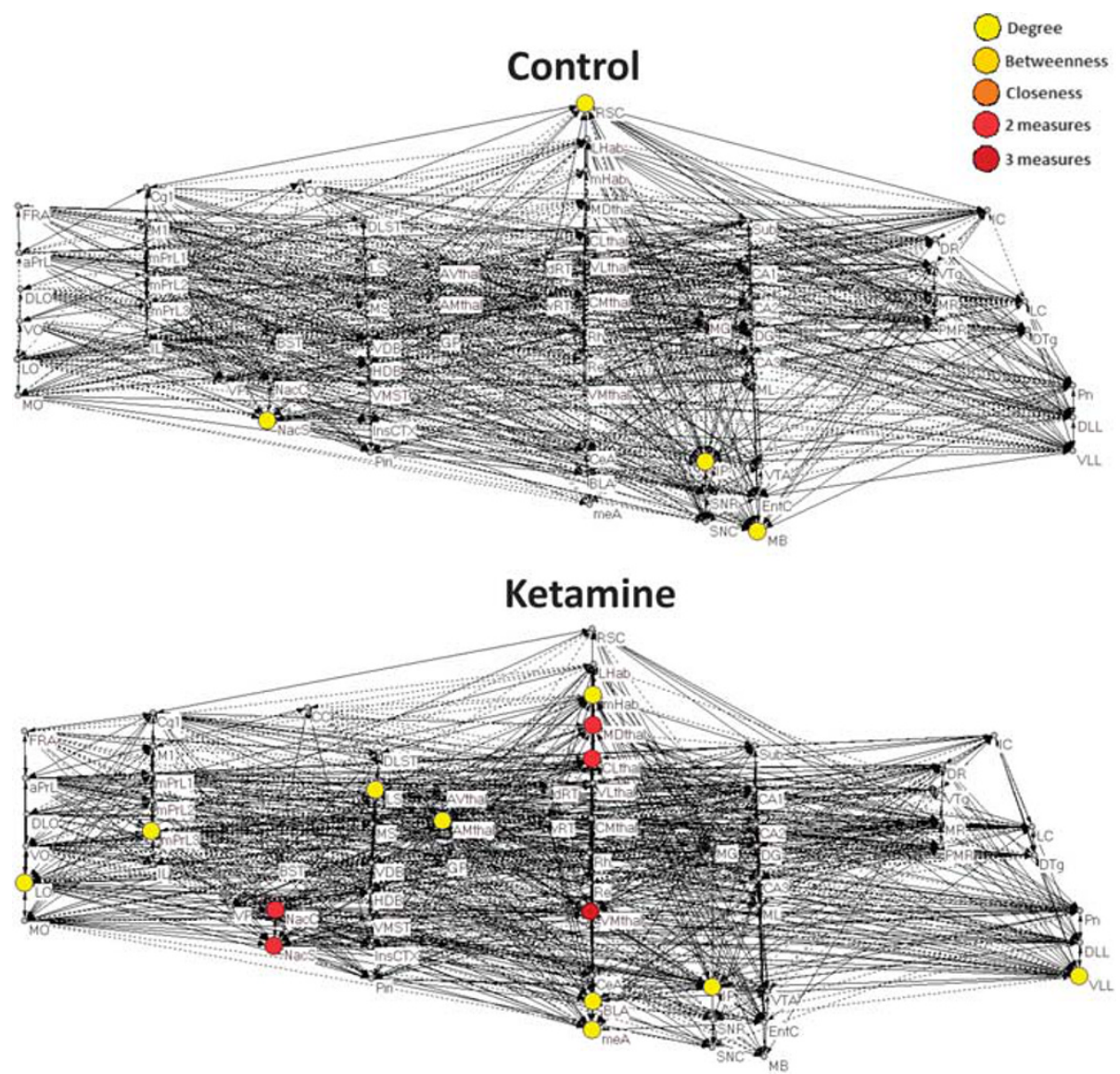

Figure 3 Hub brain regions in the functional brain networks of control and ketamine-treated mice. Graph representations of the 2-DG brain networks in control (saline treated) and ketamine-treated mice. Brain networks are shown at the 0.4 correlation threshold $(T=0.4)$. Solid edges between nodes represent a positive correlation in cerebral metabolism between brain regions, whereas broken connections (edges) denote a negative correlation in cerebral metabolism between two brain regions. If regional centrality, for any centrality measure, surpassed the $z>1.96$ threshold, calculated in comparison with I I 000 calibrated random Erdös-Rényi graphs, across the entire correlation threshold range $(T=0.3-0.4)$, then that region was considered to be a hub in the functional brain network. Large nodes are those that are defined as important hubs in the network. Node color denotes the centrality measure in which a brain region was considered to be an important hub in the brain network. These graphs were generated using the Pajek software (http:// pajek.imfm.si/doku.php?id=download).

and cortex subsystems $(p=0.040)$ was found in ketaminetreated animals that was not present in the brain network of control animals.

\section{DISCUSSION}

Through the application of established and recently validated network science algorithms we have gained important new insight into how acute NMDA receptor blockade impacts on brain functioning at a systems level. Importantly, ketamine-induced alterations in the global properties of functional brain networks (supporting enhanced connectivity) do not parallel those seen in schizophrenia patients (Micheloyannis et al, 2006; Liu et al, 2008; Lynall et al, 2010) that overall support widespread functional dysconnectivity in the disorder. In particular, our data support a ketamine-induced enhancement of prefrontal, thalamic, and amygdala regional functional connectivity, as determined by centrality analysis (Table 1; Figure 3). Furthermore, our analysis of ketamine-induced alterations in bipartite neural subsystem interactions suggests that the connectivity of these, and other, neural subsystems is profoundly altered as a result of acute ketamine treatment.

Functional brain networks in chronic schizophrenia characteristically have fewer connections (reduced mean degree $<k>$ ), a decreased efficiency for global information transfer (increased average path length, $L_{\mathrm{p}}$ ) and decreased local connectivity (decreased mean clustering, $C_{\mathrm{p}}$; Micheloyannis et al, 2006; Liu et al, 2008; Lynall et al, 2010). By contrast, we have shown that acute ketamine treatment results in a functional brain network with increased connectivity, as evidenced by an increased number of functional connections (Figure 2a, increased mean degree, $\langle k>$ ), and increased local connectivity (Figure 2c, increased mean clustering coefficient, $C_{\mathrm{p}}$ ). While the functional connectivity measure utilized in our study is derived from regional metabolic activity across an extended time period, rather than in real time as derived in human brain imaging studies, the parallels between the network connectivity alterations detected using our approach in mice and that reported in recent human brain imaging studies suggest that this is a valid approach. For example, the increased functional connectivity found in brain 
Table I Altered Hub Brain Regions in the Functional Brain Networks of Control and Ketamine-Treated Mice

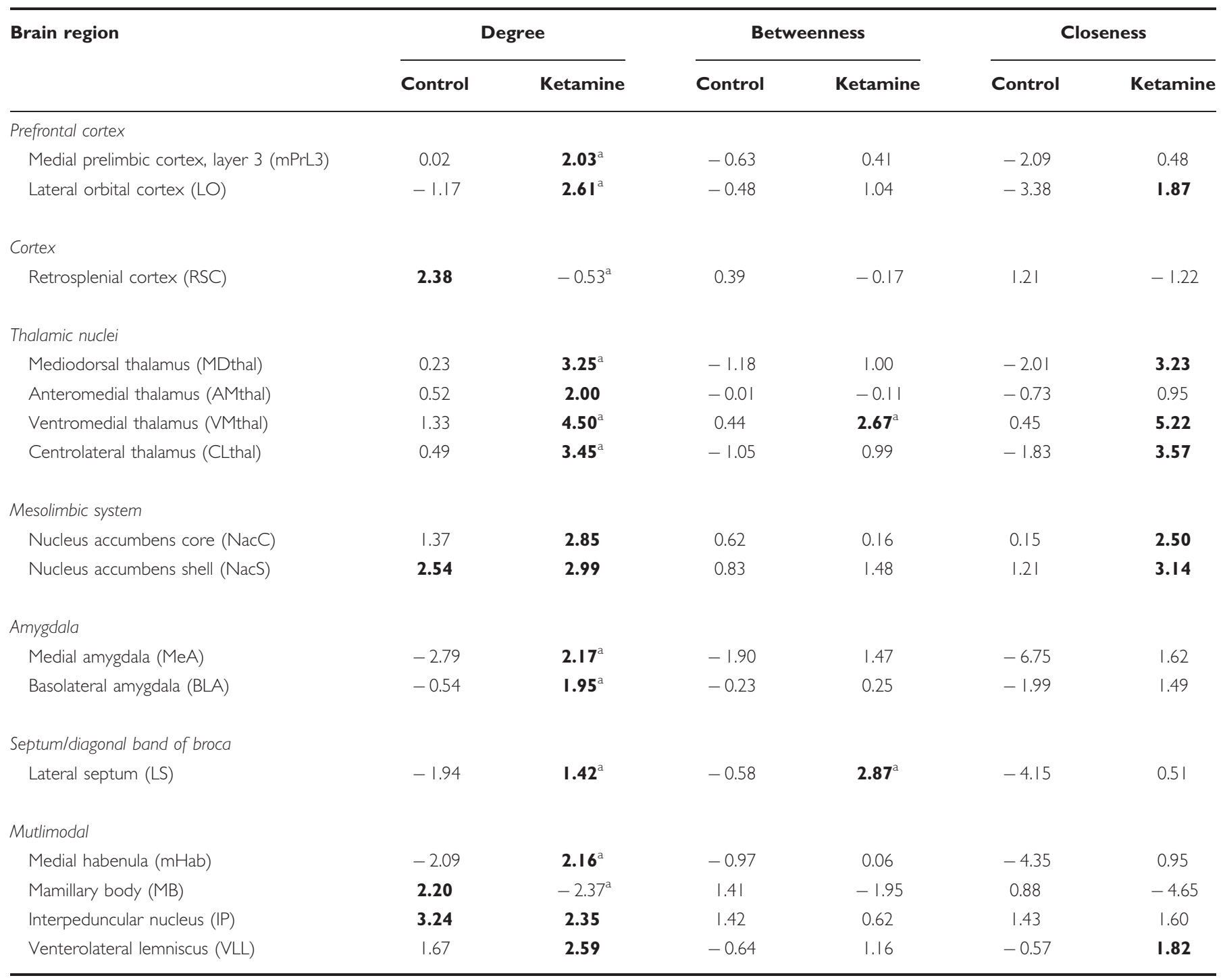

Data shown as $z$-score for each centrality measure (degree, betweenness, and closeness) for each hub brain region in the brain networks of control and ketaminetreated animals. Values in bold denote centrality measures in which a given brain region reaches the defined criteria ( $z$-score $>1.96$ relative to II 000 calibrated random Erdös-Rényi graphs across the entire correlation threshold range $(T=0.3-0.4))$ to be considered as a hub.

${ }^{a}$ Denotes $p<0.01$ significant difference in a given centrality measure between ketamine-treated and control animals (determined by comparison of the difference in real brain networks with that in I 000 random permutations of the data across the entire correlation threshold range $(T=0.3-0.4)$ with Bonferroni post hoc correction for multiple comparisons). Full data are shown in Supplementary Table S2A-D.

networks of mice treated with ketamine in our study parallels the increased functional connectivity found in the brain of healthy humans treated with subanesthetic ketamine (Driesen et al, 2013). These data suggest that, at a systems level, the mechanisms through which acute ketamine treatment induces schizophrenia-like symptoms may be profoundly divergent from those that contribute to these symptoms in the disorder. This suggestion is also consistent with the alterations we have identified at the level of regional connectivity, as determined through centrality analysis, which supports both enhanced PFC and thalamic functional connectivity. Therefore, our data suggest that the disruption of PFC-dependent cognitive processes by subanestetic ketamine treatment (Krystal et al, 2000; Nikiforuk et al, 2010; Moghaddam et al, 1997) results not from PFC functional dysconnectivity, but may result from abnormally increased PFC functional connectivity, a suggestion consistent with our previous observation that ketamine treatment enhances functional connectivity between subfields of the PFC (Dawson et al, 2013). Decreased PFC functional connectivity is widely reported in schizophrenia (Deserno et al, 2012; Honey et al, 2005; Spence et al, 2000; Zhou et al, 2007), although some studies have identified regionally selective increases in PFC connectivity in the disorder (Schlösser et al, 2003; Klingner et al, 2013). Overall, our data suggest that rather than impeding the capability of the PFC to receive, integrate, and output relevant information to other neural subsystems, through PFC dysconnectivity as seen in schizophrenia, acute ketamine treatment may decreases the signal-to-noise ratio 

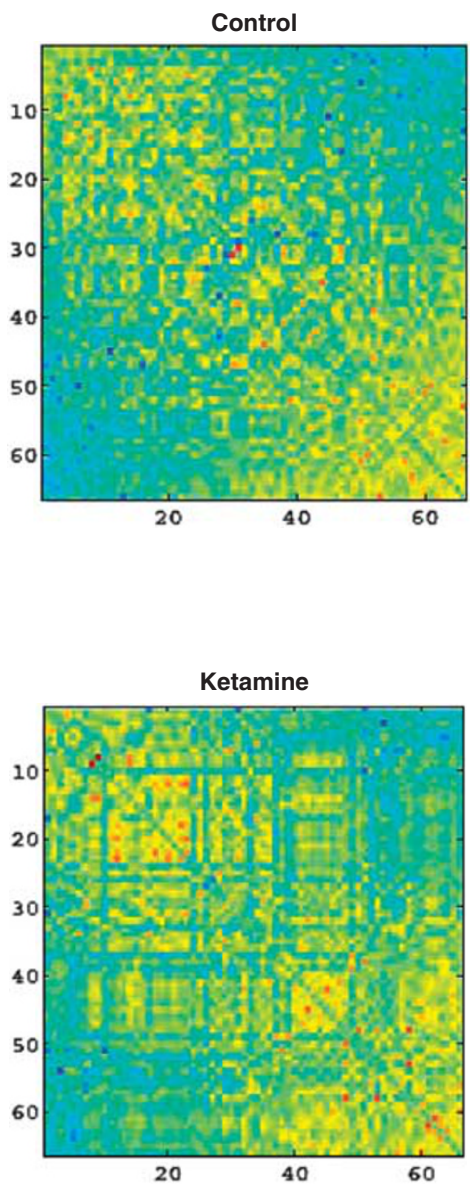

b

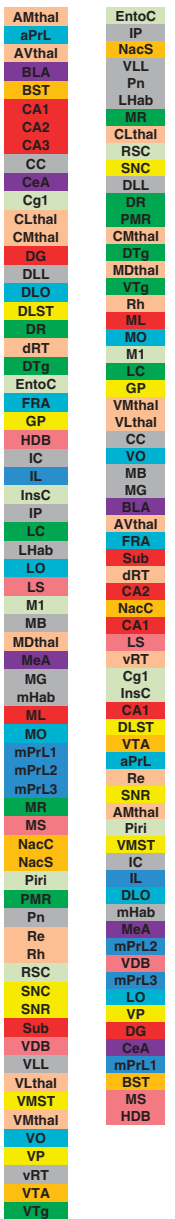

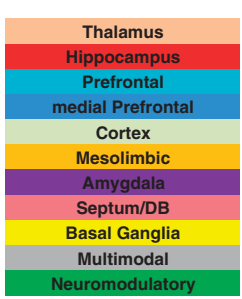

C

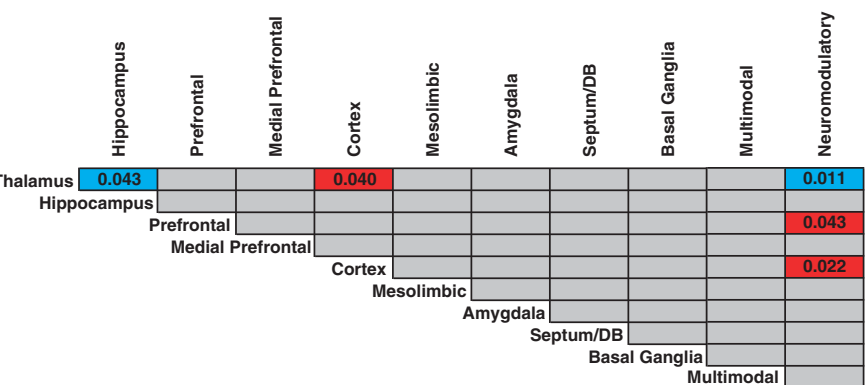

Figure 4 Ketamine-induced alterations in bipartite neural subsystem interactions. (a) Heatmaps showing the clustering of neural subsystems in control and ketamine-treated animals brain network matrices following GSVD reordering. Warm colors (red/orange) represent high/positive functional correlations between brain regions and cold colors (blue/green) represent low/negative correlations between brain regions. The Control reordered matrix identified clusters of brain regions present in the control group not present in ketamine-treated animals, and the ketamine ordered matrix shows clusters present in ketamine-treated animals not present in controls. (b) Brain region lists showing the order of brain regions in the original (alphabetical) and GSVD reordered matrices for ketamine-treated and control animals. (c) Summary diagram of significant neural subsystem bipartite interactions seen in the GSVD reordered matrices of control but not ketamine-treated animals (defined in blue) and those seen in ketamine-treated but not in control animals (defined in red). Values indicate the significance level of the given bipartite neural subsystem interaction, determined by comparison of the joint variance of each bipartite neural subsystem in the real GSVD reordered matrices as compared with that seen in 10000 random permutations of the real data. These $p$-values were adjusted post hoc by Bonferroni-Holm correction for multiple ( I I0) comparisons. Significance is set at $p<0.05$. Full data are shown in Supplementary Table S3A and B).

of information received by the PFC, by the promotion of an abnormally increased functional connectivity of this neural subsystem. This suggested mechanism is not only consistent with recent human brain imaging data showing that subanesthetic ketamine treatment increases global brain functional connectivity (Driesen et al, 2013), but is also consistent with emerging electophysiological data supporting a reduced signal-to-noise after ketamine treatment in the hippocampal CA3 subfield (Saunders et al, 2011) and of gamma oscillations in the somatosensory thalamocortical system (Kulikova et al, 2012) of rodents. Our data suggest that similar mechanisms may occur in the PFC, where ketamine is known to directly increase the amplitude of gamma oscillations through the blockade of local NMDA receptors (McNally et al, 2011) and where the NMDA receptor antagonist MK-801 has been shown to disrupt the firing pattern of PFC neurons (Jackson et al, 2004). Interestingly, our recent data show that prolonged NMDA receptor hypofunction, as induced by repeated subchronic phencyclidine treatment in rats, is capable of inducing the functional network dysconnectivity characteristic of chronic schizophrenia (Dawson et al, 2014). This suggests that plasticity-dependent modifications in brain circuitry that result from prolonged but not from acute NMDA receptor hypofunction, such as decreased expression of parvalbumin in GABAergic neurones (Cochran et al, 2003) and altered neurotransmitter receptor expression (Steward et al, 2004), are essential to recapitulate the alterations in functional brain networks seen in chronic schizophrenia. Moreover, sustained NMDA receptor hypofunction induces the 
hypofrontality characteristic of the disorder (Cochran et al, 2003; Dawson et al, 2012, 2014), whereas acute ketamine treatment induces a contrasting hyperfrontality, a paradox that is yet to be resolved given the schizophrenia-like symptoms induced by acute ketamine treatment. It may be the case that ketamine-induced alterations in functional brain network structure have the greatest translational relevance to those symptoms present during acute psychotic episodes rather than those that remain present in chronic schizophrenia patients treated with antipsychotics. That is to say that ketamine-induced alterations in functional brain networks may more accurately model the state-dependent alterations in functional brain connectivity that are present during an acute psychotic episode, rather than the trait alterations in functional brain network connectivity evident in chronic schizophrenia, which may be masked during acute psychosis. The suggestion that decreased brain network connectivity may be a trait of brain networks in schizophrenia is supported by recent evidence showing that individuals with an increased risk for the disorder show altered brain network properties characteristic of schizophrenia (Li et al, 2012), again supporting network dysconnectivity. However, as the properties of functional brain networks during acute psychosis are yet to be characterized, the possibility that state-dependent alterations in brain network connectivity during acute psychosis may mask those evident as a trait of the disorder remains speculative. The characterization of functional brain network connectivity in schizophrenic individuals during their first episode of acute psychosis and subsequently when stabilized by antipsychotic treatment would be an essential step in testing this hypothesis, and also in further resolving the true translational relevance of subanethetic ketamine treatment. The suggestion that subanesthetic ketamine treatment may have greater translational relevance to at least some of the cognitive deficit seen in the disorder that are evident during the early stages of the disease but not in chronic schizophrenia is supported by recent data (Hauser et al, 2011; Moore et al, 2013).

Our data also support thalamic hyperconnectivity in the brain networks of animals treated with ketamine. This is consistent with our previous observation that acute ketamine treatment enhances connectivity between the thalamic nuclei (Dawson et al, 2013). This contrasts with the thalamocortical dysconnectivity widely reported in schizophrenia, which is particularly evident in terms of PFC-thalamic dysconnectivity (Marenco et al, 2012; Welsh et al, 2010; Zhou et al, 2007; Woodward et al, 2012), although this is not always found (Klingner et al, 2013; Schlösser et al, 2003). In addition, there are examples of enhanced thalamic connectivity to other cortical subfields in schizophrenia, such as to motor and somatosensory areas (Woodward et al, 2012) and to the insular cortex (CorradiDell'Acqua et al, 2012). Interestingly, we did find evidence for enhanced thalamic connectivity to cortical regions (Figure 4c), with the exception of the PFC, following ketamine treatment in this study. Our previous data, and data from other groups, suggest that the direct actions of ketamine in the reticular thalamus (RT) may be a key mechanism through which ketamine promotes the abnormal connectivity of other thalamic regions, in part through the disinhibition of thalamocortical projections (Dawson et al, 2013; Zhang et al, 2009). Given that the cognitive and functional roles of specific thalamic nuclei and their cortical connections are poorly defined, with the exception of the PFC-thalamic connection, the potential contribution of these alterations to ketamine's ability to model schizophrenia certainly warrants further systematic investigation. Clearly, our data suggest a disconnection between many of the systems level alterations induced by acute ketamine treatment and those seen in the brain of schizophrenia patients and thus these must be more clearly resolved to more rigorously define the specific translational value of this model. Understanding the plasticity alterations that occur in the brain as a result of repeated NMDA receptor blockade may be particularly important in elucidating the contribution of this mechanism to the alterations in brain network connectivity seen in schizophrenia.

For the first time, we have shown that bipartite functional interactions between distinct neural subsystems are dramatically altered as a result of acute NMDA receptor blockade. In particular, the functional connectivity of the PFC, thalamus, HP, and neuromodulatory neural subsystems is significantly altered by ketamine treatment. The ketamineinduced alterations we have shown in the bipartite connectivity of these neural subsystems, as determined by GSVD analysis, parallel those we have previously reported using regional functional connectivity analysis (Dawson et al, 2013), that included a ketamine-induced enhancement of functional connectivity between selected neuromodulatory nuclei (DR and LC) and the PFC. As the neuromodulatory nuclei defined in this study included both the DR and the MR, sources of serotonergic innervation to the PFC, and the LC, a primary source of noradrenergic innervation to the PFC, the enhanced connectivity between these neural subsystems is consistent with the known ability of acute ketamine to profoundly enhance both serotonin (Lindefors et al, 1997; Amargos-Bosch et al, 2006) and noradrenaline levels in the PFC (Kubota et al, 1999). The altered connectivity of these neuromodulatory neurotransmitter systems may directly contribute to the disruption of PFCdependent processes by acute NMDA receptor blockade, a suggestion supported by the observation that the application of antagonist for the receptors of these neuromodulatory systems can attenuate the disruptive effects of NMDA receptor antagonists on $\mathrm{PFC}$-dependent cognitive processes (Metzer et al, 2011; Mirjana et al, 2004), PFC physiology (Jentsch et al, 1998), and other translationally relevant behaviors (Bakshi and Geyer, 1997; Stuchlik et al, 2009). Overall, our data suggest that the connectivity of the neuromodulatory subsystem to the PFC is profoundly altered by acute ketamine treatment and is likely to contribute to ketamine's disruptive effects on PFC-dependent cognitive processes. In contrast to the PFC, we found that the bipartite functional connectivity of the thalamus to the neuromodulatory subsystem and HP was decreased, whereas thalamic connectivity to the cortical subsystems was increased by acute ketamine treatment. The direct inhibition of thalamic GABAergic neurons, consistent with the decreased metabolic demand of these nuclei (Figure 1, as 2-DG imaging largely reflects the metabolic demands of localized synapses), by NMDA receptor blockade (Zhang et al, 2009) may underlie the functional dysconnectivity of this neural subsystem from that of the neuromodulatory 
nuclei. In addition this would lead to the disinhibition of thalamic glutamatergic projections to the cortex and other neural subsystems (Sharp et al, 2001), a suggestion consistent with the enhanced bipartite thalamus-cortex connectivity also seen in ketamine-treated animals.

Overall, our data suggest that the systems level alterations induced by subanesthetic ketamine treatment are distinct from those seen in chronic schizophrenia. These, along with other data, suggest that plasticity-dependent alterations in brain circuitry that result from chronic but not from acute NMDA receptor blockade most accurately reflect the systems level differences in brain network functioning seen in schizophrenia. Characterizing these plasticity mechanisms may provide key insight into the transition from the acute psychotic to the chronic disease state in schizophrenia, and could provide opportunities for the development of novel treatment interventions. In addition, these data provide dramatic new insight into the altered brain functioning, and the altered interactions between defined neural subsystems, that result from acute NMDA receptor blockade.

\section{FUNDING AND DISCLOSURE}

This work was supported by the Psychiatric Research Institute of Neuroscience in Glasgow (PsyRING to ND), a joint initiative between the Universities of Glasgow and Strathclyde and the National Health Service of Greater Glasgow and Clyde; the Engineering and Physical Sciences Research Council Bridging the Gap Program (grant number EP/E018858/1 to ND, JAP, and DJH); the Engineering and Physical Research Council (grant number EP/E049370/1 to $\mathrm{DJH}$ ) and the Medical Research Council (grant number G0601353 to DJH). MD is supported by the Engineering and Physical Research Council funded doctoral training grant in medical devices. The authors declare no conflict of interest.

\section{REFERENCES}

Amargos-Bosch M, Lopez-Gill X, Artigas F, Adell A (2006). Clozapine and olanzapine, but not haloperidol, suppress serotonin efflux in the medial prefrontal cortex elicited by phencyclidine and ketamine. Int J Neuropsychopharmacol 9: 565-573.

Bakshi VP, Geyer MA (1997). Phencyclidine induced deficits in prepulse inhibition of startle are blocked by prazosin, an alpha-1 noradrenergic antagonist. J Pharmacol Exp Ther 283: 666-674.

Bassett DS, Bullmore E, Verchinski BA, Mattay VS, Weinberger DR, Meyer-Lindenberg AM (2008). Hierarchical organization of human cortical networks in health and schizophrenia. J Neurosci 28: 9239-9248.

Benetti S, Mechelli A, Picchioni M, Broome M, Williams S, McGuire P (2009). Functional integration between the posterior hippocampus and prefrontal cortex is impaired in both first episode schizophrenia and the at risk mental state. Brain 132: 2426-2436.

Bifone A, Gozzi A, Schwarz AJ (2010). Functional connectivity in the rat brain: a complex network approach. Magn Reson Imaging 28: $1200-1209$.

Chih-Liang C, Upadhyay J, Marek GJ, Baker SJ, Zhang M, Mezler M et al (2011). Awake rat pharmacological MRI as a translational pharmacodynamic biomarker: mGluR2/3 agonist modulation of ketamine-induced BOLD signals. J Pharmacol Exp Ther 3: 709-715.
Cochran SM, Kennedy M, McKerchar CE, Steward LJ, Pratt JA, Morris BJ (2003). Induction of metabolic hypofunction and neurochemical deficits after chronic intermittent exposure to phencyclidine: differential modulation by antipsychotic drugs. Neuropsychopharmacology 28: 265-275.

Corradi-Dell'Acqua C, Tomelleri L, Bellani M, Rambaldelli G, Cerini R, Pozzi-Mucelli R et al (2012). Thalamic-insular dysconnectivity in schizophrenia: evidence from structural equation modeling. Hum Brain Mapp 33: 740-752.

Cosgrove J, Newell T (1991). Recovery of neuropsychological function during reduction in use of phencyclidine. J Clin Psychol 47: 159-169.

Dawson N, Ferrington L, Lesch KP, Kelly PAT (2011). Cerebral metabolic responses to $5-\mathrm{HT}_{2 \mathrm{~A} / \mathrm{C}}$ receptor activation in mice with genetically modified serotonin transporter (SERT) expression. Eur Neuropsychopharm 21: 117-118.

Dawson N, Thomson RJ, McVie A, Thomson DM, Morris BJ, Pratt JA (2012). Modafinil reverses phencyclidine-induced deficits in cognitive flexibility, cerebral metabolism and functional brain connectivity. Schizophr Bull 38: 457-474.

Dawson N, Morris BJ, Pratt JA (2013). Subanaesthetic ketamine treatment alters prefrontal cortex connectivity with thalamus and ascending subcortical systems. Schizophr Bull 39: 366-377.

Dawson N, Xiao X, McDonald M, Higham DJ, Morris BJ, Pratt JA (2014). Sustained NMDA receptor hypofunction induces compromised neural systems integration and schizophrenia-like alterations in functional brain networks. Cereb Cortex 24: 452-464.

De la Fuente-Sandoval C, Leon-Ortiz P, Favila R, Stephano S, Mamo D, Ramirez-Bermudez J et al (2011). Higher levels of glutamate in the associative striatum of subjects with prodromal symptoms of schizophrenia and patients with first-episode psychosis. Neuropsychopharmacology 36: 1781-1791.

Deserno L, Sterzer P, Wustenberg T, Heinz A, Schladenhauf F (2012). Reduced prefrontal-parietal effective connectivity and working memory deficits in schizophrenia. J Neurosci 32: 12-20.

D'Souza DC, Ahn K, Bhakta S, Elander J, Singh N, Nadim H et al (2012). Nicotine fails to attenuate ketamine-induced cognitive deficits and negative and positive symptoms in Humans: implications for schizophrenia. Biol Psychiatry 72: 785-794.

Driesen NR, McCarthy G, Bhagwagar Z, Bloch M, Calhoun V, D'Souza DC et al (2013). Relationship of resting brain hyperconnectivity and schizophrenia-like synptoms produced by the NMDA receptor antagonist ketamine. Mol Psychiatry 18: 1199-1204.

Duncan GE, Miyamoto S, Leipzig JN, Lieberman JA (1999). Comparison of brain metabolic activity patterns induced by ketamine, MK-801 and amphetamine in rats: support for NMDA receptor involvement in responses to subanaesthetic dose of ketamine. Brain Res 843: 171-183.

Galci R, Boggs JD, Miller KL, Bonaventure P, Atack JR. (2008). Effects of SB-269970, a 5-HT7 receptor agonist, in mouse models predictive of antipsychotic-like activity. Behav Pharmacol 19: 153-159.

Hauser M, Moore JW, de Millas W, Gallinat J, Heinz A, Haggard P et al (2011). Sense of agency is altered in patients with putative psychotic prodrome. Schizophr Res 126: 20-27.

Hill K, Mann L, Laws KR, Stephenson CME, Nimmo-Smith I, McKenna PJ (2004). Hypofrontality in schizophrenia: a metaanalysis of functional imaging studies. Acta Psychiatr Scand 110: 243-256.

Honey GD, Pomarol-Clotet E, Corlett PR, Honey RAE, McKenna PJ, Bullmore ET et al (2005). Functional dysconnectivity in schizophrenia associated with attentional modulation of motor function. Brain 128: 2597-2611.

Irifune M, Shimizo T, Nomoto M. (1991). Ketamine-induced hyperlocomotion associated with alteration of presynaptic components of dopamine neurones in the nucleus accumbens of mice. Pharmacol Biochem Behav 40: 399-407. 
Jackson ME, Homayoun H, Moghaddam B (2004). NMDA receptor hypofunction produces concomitant firing rate potentiation and burst activity reduction in the prefrontal cortex. Proc Natl Acad Sci USA 101: 8467-8472.

Jentsch JD, Wise A, Katz Z, Roth RH (1998). Alpha-adrenergic receptor modulation of the phencyclinde and delta9-tetrahydrocannabinol induced increases in dopamine utilization in the rat prefrontal cortex. Synapse 28: 21-26.

Kim JJ, Seok JH, Park HJ, Lee DS, Lee MC, Kwon LS (2005). Functional disconnection of the semantic networks in schizophrenia. Neuroreport 16: 355-359.

Klingner CM, Langbein K, Dietzek M, Smesny S, Witte OW, Sauer $\mathrm{H}$ et al (2013). Thalamocortical connectivity during resting state in schizophrenia. Eur Arch Psychiatry Clin Neurosci; e-pub ahead of print 27 July 2013. doi:10.1007/s00406-013-0417-0.

Kos T, Popik P, Pietraszek M, Schafer S, Danysz W, Dravolina O et al (2006). Effect of the 5-HT3 receptor antagonist MDL 72222 on behaviours induced by ketamine in rats and mice. Eur Neuropsychopharm 16: 297-310.

Krystal JH, Bennett A, Abi-Saab D, Belger A, Karper LP, D'Souza DC et al (2000). Dissociation of ketamine effects on rule acquisition and rule implementation: possible relevance to NMDA receptor contributions to executive cognitive functions. Biol Psychiatry 47: 137-143.

Krystal JH, Karper LP, Seibyl JP, Freeman GK, Delaney R, Bremner JD et al (1994). Subanaesthetic effects of the noncompetitive NMDA anatagonsit, ketamine, in humans: psychotomimetic, perceptual, cognitive and neuroendocrine responses. Arch Gen Psychiatry. 51: 199-214.

Kubota T, Hirota K, Yoshida H et al (1999). Effects of sedatives on noradrenaline release from the medial prefrontal cortex in rats. Psychopharmacology 146: 335-338.

Kulikova SP, Tolmacheva EA, Anderson P, Gaudias J, Adams BE, Zheng $\mathrm{T}$ et al (2012). Opposite effects of ketamine and deep brain stimulation on rat thalamocortical information processing. Eur J Neurosci 36: 3407-3419.

Lahti AC, Weiler MA, Michaelidis T, Parwani A, Tamminga CA (2001). Effects of ketamine in normal and schizophrenic volunteers. Neuropsychopharmacology 25: 455-467.

Langsjo JW, Salmi E, Kaisti KK, Aalto S, Hinkka S, Aantaa R et al (2004). Effects of subanaesthetic ketamine on regional cerebral glucose metabolism in humans. Anesthesiology 100: 1065-1071.

Li Y, Liu B, Qin W, Wang D, Yu C, Jiang T (2012). Less efficient information transfer in Cys-Allele carrier of DISC1: a brain network study based on diffusion MRI. Cereb Cortex 23: $1715-1723$.

Lindefors N, Barati S, O'Connor WT (1997). Differential effects of single and repeated ketamine administration on dopamine, serotonin and GABA transmission in rat medial prefrontal cortex. Brain Res 759: 205-212.

Liu Y, Liang M, Zhou Y, He Y, Hao Y, Song M et al (2008). Disrupted small world networks in schizophrenia. Brain 131: 945-961.

Lynall ME, Bassett DS, Kerwin R, McKenna PJ, Kitzbilcher M, Muller U et al (2010). Functional connectivity in brain networks in schizophrenia. J Neuroscience 30: 9477-9487.

Malhotra AK, Pinals DA, Adler CM, Elman I, Clifton A, Pickar D et al (1997). Ketamine-induced exacerbation of psychotic symptoms and cognitive impairment in neuroleptic-free schizophrenics. Neuropsychopharmacology 17: 141-150.

Marenco S, Stein JL, Savostyanova AA, Sambataro F, Tan HY, Goldman AL et al (2012). Investigation of anatomical thalamocortical connectivity and fMRI activation in schizophrenia. Neuropsychopharmacology 37: 499-507.

Marsman A, van den Heuvel MP, Klomp DWJ, Kahn RS, Luijten PR, Hulshoff Pol HE (2013). Glutamate in schizophrenia: a focused review and meta-analysis of ${ }^{1} \mathrm{H}$-MRS studies. Schizophr Bull 39: 120-129.
McCulloch J, Kelly PAT, Ford I (1982). Effect on apomorphine on the relationship between local cerebral glucose utilization and local cerebral blood flow (with an Appendix on its statistical analysis). J Cereb Blood Flow Metab 2: 487-499.

McNally JM, McCarley RW, McKenna JT, Yanagawa Y, Brown RE (2011). Complex receptor mediation of acute ketamine application on in vitro gamma oscillations in mouse prefrontal cortex: modelling gamma band oscillation abnormalities in schizophrenia. Neuroscience 199: 51-63.

Metzer H, Horiguchi M, Massey BW (2011). The role of serotonin in the NMDA receptor antagonist models of psychosis and cognitive impairment. Psychopharmacology 213: 283-305.

Meyer-Lindenberg AS, Olsen RK, Kohn P, Brown T, Egan MF, Weinberger DR et al (2005). Regionally specific disturbance of dorsolateral prefrontal-hippocampal functional connectivity in schizophrenia. Arch Gen Psychiatry 62: 379-386.

Micheloyannis S, Pachou E, Stam CJ, Breakspear M, Bitsios P, Vourkas M et al (2006). Small-world networks and disturbed functional connectivity in schizophrenia. Schizophr Res 87: 60-66.

Mirjana C, Baviera M, Invernizzi RW, Balducci C (2004). The serotonin receptor antagonist M100907 prevents impairment in attentional performance by NMDA receptor blockade in the rat prefrontal cortex. Neuropsychopharmacology 29: 1637-1647.

Miyamoto S, Leipzig JN, Lieberman JA, Duncan GE (2000). Effects of ketamine, MK-801 and amphetamine in regional brain 2deoxyglucose uptake in freely moving mice. Neuropsychopharmacology 22: 400-412.

Moghaddam B, Adams B, Verma A, Daly D (1997). Activation of glutamatergic neurotransmission by ketamine: a novel step in the pathway from NMDA receptor blockade to dopaminergic and cognitive disruptions associated with the prefrontal cortex. J Neurosci 17: 2921-2927.

Moore JW, Cambridge VC, Morgan H, Giorlando F, Adapa R, Fletcher PC (2013). Time, action and psychosis: using subjective time to investigate the effects of ketamine on sense of agency. Neuropsychologia 51: 377-384.

Morgan CJA, Mofeez A, Brandner B, Bromley L, Curran VH (2004). Acute effects of ketamine on memory systems and psychotic symptoms in healthy volunteers. Neuropsychopharmacology 29: 208-218.

Natsubori T, Inoue H, Abe O, Takano Y, Iwashiro N, Aoki Y et al (2013). Reduced frontal glutamate and glutamine and $\mathrm{N}$-acetylaspartate levels in patients with chronic schizophrenia but not those at clinical high risk for psychosis with first-episode schizophrenia. Schizophr Bull; e-pub ahead of print 10 September 2013. doi:10.1093/schbul/sbt124.

Niesters M, Khalili-Mahani N, Martini C, Aarts L, van Gerven J, van Buchem $M$ et al (2012). Effects of subanaesthetic ketamine on intrinsic functional brain connectivity: a placebo-controlled functional magnetic resonance imaging study in health male volunteers. Anaesthesiology 117: 868-877.

Nikiforuk A, Golembiowska K, Popik P (2010). Mazindol attenuates ketamine-induced cognitive deficits in the attentional set shifting task in rats. Eur Neuropsychopharmacol 20: 37-48.

Paxinos G, Franklin KBJ (2001). The Mouse Brain in Stereotaxic Coordinates. 2nd edn. Academic Press: London, UK.

Pitsikas N, Boultadakis A, Sakellaridis N (2008). Effects of subanaesthetic doses of ketamine on rats' spatial and nonspatial recognition memory. Neuroscience 154: 454-460.

Roberts BM, Holden DE, Shaffer CL, Seymour PA, Menniti FS, Schmidt CJ et al (2010). Prevention of ketamine-induced working memory impairments by AMPA potentiators in a nonhuman primate model of cognitive dysfunction. Behav Brain Res 212: 41-48.

Saunders JA, Gandal MJ, Siegel SJ (2011). NMDA antagonists recreate signal-to-noise ratio and timing perturbations present in schizophrenia. Neurobiol Dis 46: 93-100. 
Sharp FR, Tomitaka M, Bernaudin M, Tomitaka S (2001). Psychosis: pathological activation of limbic thalamocortical circuits by psychotomimetics and schizophrenia. Trends Neurosci 24: 330-334.

Schlösser R, Gesierich T, Kaufmann B, Vucurevic G, Hunsche S, Gawehn J et al (2003). Altered effective connectivity during working memory performance in schizophrenia: a study with fMRI and structural equation modeling. Neuroimage 19: 751-763.

Skoblenick K, Everling S (2012). NMDA antagonist ketamine reduces task selectivity in macaque dorsolateral prefrontal neurons and impairs performance of randomly interleaved prosaccades and antisaccades. J Neurosci 29: 12018-12027.

Spence SA, Grasby PM, Liddle PF, Stefan MD, Sharma T, Murray RM et al (2000). Functional anatomy of verbal fluency in people with schizophrenia and those at genetic risk. Br J Psychiatry 176: 52-60.

Steward LJ, Kennedy MD, Morris BJ, Pratt JA (2004). The atypical antipsychotic drug clozapine enhances chronic PCP-induced regulation of prefrontal 5-HT2A receptors. Neuropharmacology 47: 527-537.

Stuchlik A, Petrasek T, Vales K (2009). Effect of alpha1-adrenergic antagonist prazosin in behavioural alterations induced by MK801 in a spatial memory task in long evens rats. Physiol Res 58: 733-740.

Verma A, Moghaddam B. (1996). NMDA receptor antagonists impair prefrontal cortex function as assessed via spatial delayed alternation performance in rats: modulation by dopamine. J Neurosci 16: 373-379.

Vollenweider FX, Leenders KL, Scharfetter C, Antonini A, Maguire P, Missimer J et al (1997a). Metabolic hyperfrontality and psychopathology in the ketamine model of psychosis using positron emission tomography (PET) and [18F]fluorodeoxyglucose (FDG). Eur Neuropsychopharmacol 7: 9-24.

Vollenweider FX, Leenders KL, Oye I, Hell D, Angst J (1997b). Differential psychopathology and patterns of cerebral glucose utilization produced by (S)- and (R)-ketamine in healthy volunteers using positron emission tomography (PET). Eur Neuropsychopharmacol 7: 25-38.

Welsh RC, Chen AC, Taylor SF (2010). Low-frequency BOLD fluctuations demonstrate altered thalamocortical connectivity in schizophrenia. Schizophr Bull 36: 713-722.

Woodward ND, Karbasforoushan H, Heckers S (2012). Thalamocortical dysconnectivity in schizophrenia. Am J Psychiatry 169: 1092-1099.

Xiao X, Dawson N, MacIntyre L, Morris BJ, Pratt JA, Watson DG et al (2011). Exploring metabolic pathway disruption in the subchronic phencyclidine model of schizophrenia with the Generalized Singular Value Decomposition. BMC Syst Biol 5: 72 .

Yang SY, Hong CJ, Huang YH, Tsai SJ (2010). The effects of glycine transporter I inhibitor, N-methylglycine (sarcosine), on ketamine-induced alterations in sensorimotor gating and regional brain c-Fos expression in rats. Neurosci Lett 469: 127-130.

Zhang Y, Llinas RR, Lisman JE (2009). Inhibition of NMDARs in the nucleus reticularis of the thalamus produces delta frequency bursting. Front Neural Circuits 3: 20.

Zhou Y, Liang M, Jiang T, Tian L, Liu Y, Liu Z et al (2007). Functional dysconnecitivity of the dorsolateral prefrontal cortex in first-episode schizophrenia using resting-state fMRI. Neurosci Lett 417: 297-302.

Supplementary Information accompanies the paper on the Neuropsychopharmacology website (http://www.nature.com/npp) 